\title{
Erratum to: Yolk sac tumour: a rare cause of raised serum alpha-foetoprotein in a young child with a large liver mass
}

\author{
Annemieke S. Littooij ${ }^{1} \cdot$ Kieran McHugh $^{2} \cdot$ M. Beth McCarville $^{3} \cdot$ Neil J. Sebire $^{4}$. \\ Armita Bahrami $^{5}$ - Derek J. Roebuck ${ }^{2}$
}

Published online: 24 March 2017

(C) Springer-Verlag Berlin Heidelberg 2017

Erratum to: Pediatr Radiol (2014) 44:18-22

DOI 10.1007/s00247-013-2780-4

Dr. Bahrami's given name is misspelled and should be "Armita," as shown above.

The online version of the original article can be found at doi: http://dx.doi. org/10.1007/s00247-013-2780-4

$\triangle$ Annemieke S. Littooij alittooij@hotmail.com

1 Department of Radiology,

University Medical Center Utrecht,

Utrecht, The Netherlands

2 Department of Radiology,

Great Ormond Street Hospital for Children,

London, UK

3 Department of Radiological Sciences,

St. Jude Children's Research Hospital,

Memphis, USA

4 Department of Pathology,

Great Ormond Street Hospital for Children,

London, UK

5 Department of Pathology,

St. Jude Children's Research Hospital,

Memphis, USA 